Mini Review

\title{
Hippocampal insulin signaling and neuroprotection mediated by physical exercise in Alzheimer's Disease
}

\author{
Gabriel Keine Kuga \\ Universidade Estadual Paulista “Júlio de Mesquita Filho”, Rio Claro, SP, Brasil \\ José Diego Botezelli \\ Rafael Calais Gaspar \\ Universidade Estadual de Campinas, Limeira, SP, Brasil \\ Ricardo José Gomes \\ Universidade Federal de São Paulo, Santos, SP, Brasil \\ José Rodrigo Pauli \\ Universidade Estadual de Campinas, Limeira, SP, Brasil \\ José Alexandre Curiacos de Almeida Leme \\ Centro Universitário Auxilium Unisaleasiano, Lins, SP, Brasil
}

\begin{abstract}
Epidemiological studies indicate continuous increases in the prevalence of Alzheimer's Disease (AD) in the next few decades. The key feature of this disease is hippocampal neurodegeneration. This structure has an important role in learning and memory. Intense research efforts have sought to elucidate neuroprotective mechanisms responsible for hippocampal integrity. Insulin signaling seems to be a very promising pathway for the prevention and treatment of $\mathrm{AD}$. This hormone has been described as a powerful activator of neuronal survival. Recent research showed that reduced insulin sensitivity leads to low-grade inflammation, and both phenomena are closely related to AD genesis. Concomitantly, exercise has been shown to exert anti-inflammatory effects and to promote improvement in insulin signaling in the hippocampus, which supports neuronal survival and constitutes an interesting non-pharmacological alternative for the prevention and treatment of $\mathrm{AD}$. This review examines recent advances in understanding the molecular mechanisms involved in hippocampal neuroprotection mediated by exercise.
\end{abstract}

Keywords: insulin, hippocampus, inflammation, physical exercise.

\section{Introduction}

Alzheimer's disease (AD) is the most common form of dementia manifestation ${ }^{1}$ and its prevalence increases with aging population ${ }^{2}$. Alzheimer's Disease International ${ }^{3}$ estimates that dementias reach around 46.8 million people in the world in 2015. Projections indicate that this number will double every 20 years, reaching a 74 million in 2030 and 131 million in $2050^{3}$. In 2050, it is expected that at least 100 million of these patients will suffer from $\mathrm{AD}^{4,5}$. In the last year, 818 billion dollars were invested to treat the main forms of dementia. This cost includes hospital expenses, caregivers services and personal expenses, and in 2030, it is estimated a budget of 2 trillion dollars ${ }^{3}$. Among the reasons to justify this exorbitant cost it is the absence of an effective cure or therapeutic treatment that really slows the progress of $\mathrm{AD}$ and other dementias.

Despite significant progress in elucidating the molecular mechanisms involved in neuron survival and the main agents involved in neuronal protection or neuron degeneration in the hippocampus, the scientific community still has big gaps to translate these findings across species: therapies that work in rodents often do not succeed in humans ${ }^{6}$.

In hippocampal neurons, insulin and growth factors such as BDNF (brain derived neurotrophic factor), IGF-1 and 2 (Insulinlike growth factors 1 and 2) and VEGF (vascular endothelial growth factor) transmit the intracellular signal for neuronal integrity. Under regular physiological conditions, insulin and growth factors act properly to promote neuronal, survival keeping the hippocampus functionality. When insulin and growth factors are inhibited to properly exercise its effects we have a favorable condition for the establishment of AD. Changes in insulin and growth factors signaling disrupts neuronal survival contributing to $\mathrm{AD}$ pathogenesis. The neuropathology hallmark of $A D$ is characterized by accumulation of $\beta$-amyloid, synaptic dysfunction, neuronal inflammation, phosphorylation of tau protein and neurofibrillary tangle formation ${ }^{1,6}$.

Exercise has been shown to attenuate inflammation in the hippocampus? ${ }^{7}$. The insulin sensitizing effects of physical exercise in peripheral tissues are well described in the literature ${ }^{8}$. However, it is more recent the progress to unveil the effects on 
the central nervous system (SNC), especially in hippocampal functions and a possible neuroprotective role in $\mathrm{AD}^{4}$. Therefore, the objective of this review is to present the molecular aspects involved in the development of $\mathrm{AD}$ and the role of physical exercise in the prevention and treatment of this disorder. This study is a narrative mini-review, including relevant work in the author's field of expertise and current literature. Articles that describe the molecular mechanisms of cellular signaling in $\mathrm{AD}$ were selected, with special attention to insulin signaling pathway. In addition, were included papers that highlight the relevant role of physical exercise to prevent and attenuate the inflammatory and neurodegenerative process of this disease.

\section{Hippocampal Insulin Signaling}

Insulin exerts intracellular signaling by binding to the insulin receptor (IR). The IR is composed of two $\alpha$-subunits and two $\beta$-subunits connected through disulfide bonds. Insulin binding to $\alpha$-subunit induces receptor conformational change and activates the tyrosine kinase activity of the $\beta$-subunit. The $\beta$ subunit has an intrinsic tyrosine kinase activity, having the ability to phosphorylate itself and other tyrosine substrates. Tyrosine phosphorylation of major insulin receptor substrates (IRS-1 and IRS-2) by IR creates binding sites for the enzyme phosphatidylinositol-3 kinase (PI3K), activating it. Once activated, PI3K converts the membrane phospholipid phosphatidylinositol 4,5bisphosphate to phosphatidylinositol 3,4,5-trisphosphate. This conversion mediates the activation of the protein kinase $\mathrm{B}(\mathrm{PKB} /$ Akt) through the 3-phosphoinositide dependent kinase (PDK). The protein kinase $\mathrm{b} \mathrm{PKB} / \mathrm{Akt}$ signaling coordinates diverse cellular responses ${ }^{8,9}$. One of the main actions of this protein in the hippocampus is to promote neuronal survival. Akt enhances phosphorylation of FOXO transcription factor (forkhead box transcriptional factor family) and promotes its extrusion from the cell nucleus for subsequent proteasomal degradation. The FOXO presence in the nucleus acts as transcription factor of pro-apoptotic proteins and contributes to degenerative process ${ }^{10}$.

In addition, Akt is responsible for inactivating protein of GSK3- $\beta$ (glycogen synthase kinase $3 \beta$ ). When activated in hippocampal neurons, GSK3- $\beta$ promotes the phosphorylation of tau protein a major contributor to AD. By reducing the ability of insulin to propagate its intracellular signal, the continuous activation of GSK3- $\beta$ promotes hyperphosphorylation of tau protein ${ }^{9}$ and the subsequent formation of neurofibrillary tangles. All this mechanism results deleterious effects on synaptic dysfunction and cognitive impairment ${ }^{1}$.

The accumulation of $\beta$-amyloid oligomers is another pathogenic aspect of AD. These oligomers are main component of senile plaques seen in brains with $\mathrm{AD}$. The $\beta$-amyloid protein is a peptide originated from cleavage of amyloid precursor protein (APP) by gamma-secretase enzyme ${ }^{11}$ and leads to neurotoxicity. Several studies have provided evidence between insulin metabolism and $\beta$-amyloid accumulation. The hyperinsulinemia may increase the extracellular concentration of $\beta$-amyloid protein by increasing its release from neurons and modulation of gamma-secretase enzyme activity ${ }^{12}$. Other findings have shown that increased insulin concentration in the extracellular medium competes with $\beta$-amyloid protein as substrate of the insulin degradation enzyme (IDE). This protein may degrade insulin or $\beta$-amyloid protein. By promoting the degradation of insulin instead of $\beta$-amyloid protein, this mechanism hits the neuron in two different ways. First, by reducing the degradation of $\beta$-amyloid protein and second, by reducing the binding of insulin IR and, thus, its ability to spread its neuroprotective signal intracellularly ${ }^{12}$.

Inside the cell, the phosphorylation of IRS 1 and 2 also activates the mitogen activated protein kinase (MAPK) cascade of Ras/MEK/ERK ${ }^{13}$, triggering multiple cell responses. The ERK phosphorylation leads growth, differentiation and proliferation of hippocampal neurons, being essential for neuronal survival and neurogenesis ${ }^{14}$ through the activation of cytosolic proteins and transcription factors in the nucleus ${ }^{15}$. The main target protein phosphorylation of ERK (extracellular signal-regulated kinase) is CREB (cAMP response binding protein). This protein function in hippocampus results the formation of long-term memory and increases synaptic plasticity ${ }^{16}$. These modifications generate activity-dependent responses of synaptic transmission efficiency (functional plasticity) and changes in the structure and number of synaptic connections (structural plasticity) ${ }^{17}$.

Understanding insulin signaling is important to understand the connection between the mechanisms underlying inflammation and the reduced action of this hormone. Moreover, this accumulated knowledge involving the insulin signal transduction in the hippocampus has allowed the understanding of the effect of exercise to cooperate in mitigating the disease.

\section{Inflammation and Insulin Resistance}

The low-grade inflammation is a molecular process that reduces the capacity of insulin to propagate their intracellular signal, culminating in a state of insulin resistance. Compensatory body's response to reduced insulin action consists of the chronic conditions of hyperinsulinemia ${ }^{18}$, providing the basis for the establishment of Diabetes Mellitus Type 2 (DM2). It was found that the $\mathrm{AD}$ has features in common with $\mathrm{DM} 2$, leading some researchers to announce the $\mathrm{AD}$ as Diabetes Type 3. The insulin resistance and decrease of growth factors are closely associated with typical neurodegeneration in $\mathrm{AD}^{9}$. During inflammation, hippocampal levels of pro-inflammatory cytokines such as TNF- $\alpha$ (tumor necrosis factor alpha) and $\beta$-IL1 (interleukin $1 \beta$ ) are elevated ${ }^{7}$. One factor that is highly related to increased cytokines is aging ${ }^{7,19}$ and this process is denominated "Inflammaging"20,21. This condition has high prevalence in the elderly population and $\mathrm{AD}$ carriers ${ }^{3}$. The cytokines bind to their receptors trigger the inflammatory pathway through the JNK protein (c-Jun N-terminal kinase) and IKK (I kappa B kinase) activation. Both proteins disrupt insulin signaling and exacerbate inflammation by increasing the transcription of inflammatory genes ${ }^{22}$.

JNK is a serine kinase activated by TNF- $\alpha$ binding to the receptor or through the activation of TLR-4 receptors (Toll-like receptor 4$)^{21}$. When activated by these pathways, the JNK promotes phosphorylation of IRS-1 serine ${ }^{307}$ turning off key steps 
of its signaling cascade and constituting a major mechanism of insulin resistance ${ }^{22}$. JNK also acts as activator of the transcription factor AP1 leading to the transcription inflammatory genes and cytokines production ${ }^{22}$. The post-mortem brains from AD patients showed increased phosphorylation of IRS-1 in the serine ${ }^{307}$ residue and JNK expression, just as like in T2DM patients ${ }^{23}$. Other studies have shown that both oligomers of $\beta$-amyloid protein $^{23}$ as a high fat diet activate JNK phosphorylation in hippocampal neurons ${ }^{24}$. Both findings led to increased IRS-1 serine ${ }^{307}$ phosphorylation and tau protein levels. This mechanism brings a strong association between $\mathrm{AD}$ and obesity due to the reduction of insulin signaling in neuronal survival. Although the aging process is associated with increased inflammation, this situation is even worse in the obese elderly.

The other mediator of insulin resistance is IKK. When activated by the signaling cascade of TNF- $\alpha$ and TLR-4, the IKK cleaves the NF-kB/I $\kappa \beta$ complex ${ }^{19}$. This complex disruption releases the transcription factor $\mathrm{NF}-\mathrm{kB}$ (nuclear factor kappa $\mathrm{B})$, which migrates to the nucleus and initiates transcription proteins such as TNF- $\alpha$, IL1- $\beta$ and iNOS ${ }^{7,19,22}$. In addition, IKK is also capable of promoting IRS- 1 serine ${ }^{307}$ phosphorylation in hippocampal neurons upon activation by oligomers of $\beta$-amyloid protein $^{23}$. The aging contributes to increased activation of NF$\mathrm{kB}$ transcription factor in hippocampus and may be one of the factors by which AD is mainly related to elderly population ${ }^{7}$.

The numerous events that lead to activation of inflammatory signaling involve formation and clearance of several different proteins. The crucial organelle for this machinery is the endoplasmic reticulum (ER). This structure is a major regulator of inflammation feedback. This important site is responsible for the proper synthesis and protein folding ${ }^{22}$. This organelle is also highly sensitive to disturbances in cellular homeostasis and ER malfunction increases the concentration of misfolded proteins, altering the normal functionality of these proteins. This state of unbalance of protein folding is denominated endoplasmic reticulum stress ${ }^{25}$. The circulating lipids has a powerful effect of endoplasmic reticulum stress, linking the excessive consumption of this macronutrient and obesity ${ }^{25}$. As cellular response to restore the level of unfolded proteins properly there is a mechanism denominated UPR (unfolded protein response). This mechanism consists in the activation of proteins IRE1 (inositol-requiring enzyme 1), PERK (protein kinase (PKR) -like endoplasmic reticulum kinase) and ATF6 (activating transcription factor 6). This regulation reduces the overall transcription of inflammatory agents and increase the synthesis of chaperones, restoring the proper function of endoplasmic reticulum ${ }^{22}$. However, with persistent ER stress, the UPR ultimately triggers cellular defense mechanisms by inflammation and programmed cell death (apoptosis) in last case. This role process involves the previous described proteins JNK and NF-kB.

In summary, these mechanisms (JNK, IKK and ER stress) are the major agents of low-grade inflammation and insulin resistance. Activation of these pathways in the hippocampus results in reduced ability of insulin to exerts its neuroprotective effect and the consequences are accumulation of $\beta$-amyloid oligomers, hyperphosphorylation of tau protein and neuronal apoptosis, creating favorable conditions for the genesis of AD.
The next step is to discover how exercise works in this context to reduce the expression and activity of inflammatory and apoptotic proteins.

\section{Neuroprotection Mediated by Physical Exercise}

The effects of exercise on insulin signaling pathway have been intensively investigated in the last few years. On peripheral tissues, exercise stimulates the glucose uptake independent of insulin via activation of protein kinase activated by AMP (AMPK) and mTOR (mammalian target of rapamycin $)^{26}$. In addition, the exercise reduces the activation of serine kinase $\mathrm{JNK}$ and $\mathrm{IKK}^{27}$, attenuating the subclinical inflammation and the deleterious effects pro-inflammatory cytokines on the insulin signal transduction. However, physical exercise exerts different effects on the CNS. Several beneficial results demonstrated that physical exercise improves synaptic plasticity and cognition ${ }^{13,28}$, regulates the production and degradation of neurotransmitters ${ }^{29,30}$ and increases angiogenesis ${ }^{31}$ and neurogenesis ${ }^{32}$. Although the molecular mechanisms responsible for these events are not completely understood, there are strong evidences showing the exercise mediating the production and sensitivity to growth factors such as BDNF, IGF-1, IGF-2 and VEGF $1,28,32,33$.

Diegues and colleagues ${ }^{13}$ conducted an elegant study in which investigated the molecular mechanisms of physical training in diabetic Wistar rats. After induction of diabetes by administration of Alloxan, these researchers have found impaired performance in Morris water maze (spatial memory test) in sedentary diabetic rats compared to diabetic animals subjected to physical training (6 weeks of swimming, 1 hour daily for 5 days per week and the animal supporting load corresponding to $90 \%$ of the anaerobic threshold). Further analysis in hippocampus showed increased IGF-1 receptor expression and phosphorylation of Akt-1/2 isoforms in trained groups. The sedentary diabetic rats, however, presented increased $\beta$-amyloid phosphorylation. Furthermore, the exercise reduced both the expression of APP and the phosphorylation of tau protein, which is consistent evidence about the neuroprotective effects of exercise on hippocampal neurons and prevention of AD.

The hippocampal neuroprotection mediated by exercise has been under investigation of Um and colleagues ${ }^{34}$. In this remarkable study, Tg-NSE / hPS2m mice (transgenic model of AD) performed physical training for 3 months on a treadmill ( 5 days per week with a daily 60 minutes and speed of $12 \mathrm{~m} / \mathrm{min}$ ). Analyzing hippocampal samples, they found reduced expression of $\beta$-amyloid protein, Cox-2 (cyclooxygenase 2 ) and caspase- 3 (pro-apoptotic protein) in trained animals. These alterations were concomitant to reduced JNK and tau phosphorylation and increased ERK phosphorylation, CREB, PI3K, Akt and GSK3- $\beta$, all involved in insulin signaling pathway. They also found reduced apoptotic state of hippocampal neurons in mice trained through a TUNEL assay to detect DNA fragmentation typical of apoptotic process. In addition, they have found a substantial increase of BDNF expression. These results demonstrate in mice the powerful effect of exercise training to reduce or even reverse the inflammation and apoptosis of hippocampal neurons present in AD. 
The high fat diet consumption and obesity are one of the main cause of ER stress. Feeding Sprague-Dawley rats with high fat diet for 8 weeks was sufficient to induce obesity and ER stress in hippocampus ${ }^{35}$. A study conducted by Cai and colleagues ${ }^{35}$ supported this fact, revealing increased IRE- 1 and eIF2a phosphorylation/total extract protein ration in obese rats. Physical training treadmill ( 8 weeks, 5 days per week with 40 minutes daily and speed of $18 \mathrm{~m} / \mathrm{min}$ ) have shown a powerful tool in this scenario. Physical exercise recovered the ERK and CREB phosphorylation in high fat animals in comparison to sedentary chow-fed animals. In fact, the trained chow-fed animals showed the highest levels of ERK and CREB phosphorylation among all groups studied. Obesity increased expression of apoptotic proteins (caspase-12, CHOP and Bax) and reduced Bcl-2(antiapoptotic) content. However, it was significantly restored in the obese trained group in comparison to obese sedentary group, but not to levels of lean animals. While physical exercise contributed to reduction of ER stress, these results demonstrate that the losses caused by obesity are not fully restored. This gap can be an important target for development of more efficient protocols of physical training. Further, reinforcing the importance of weight control and reduced intake of saturated fats to prevent the degeneration of hippocampal neurons.

The activation of the transcription factor NF-kB appears to have close relation to aging and the AD development. Aged Wistar animals (20 months) showed increased activation of NF-kB in hippocampus compared to young animals (3 months) ${ }^{7}$. In the same study, it was investigated the effect of two weeks of exercise on a treadmill (20 minutes per day to $60 \%$ of the estimated intensity of maximum consumption of animal oxygen) on the activation of NF-kB in the hippocampus, and there was no significant difference between young animals that were or not subjected to exercise. However, in older animals (20 months), physical exercise was able to reduce the NF-kB activation in the hippocampus 1 hour after the last exercise bout. In this particular study, Wistar rats were not induced obesity, diabetes or DA, leading us to believe that exercise may attenuates the effect of aging ${ }^{7}$. Future studies shall be conducted to investigate the effects of exercise on the NF-kB activation in the hippocampus of animals at different ages.

Recent results indicate that physical exercise promotes increased phosphorylation of AMPK in the hippocampus ${ }^{36,37}$. AMPK is an essential regulator of autophagy leading degradation and inhibition of poorly folded proteins. This mechanism prevents and stops the ER stress contributing for the cell function. The pharmacological activation of AMPK in cell culture was able to promote the degradation of $\beta$-amyloid protein ${ }^{38}$. However, further researches need to investigate the AMPK activation mediated by physical exercise in vivo.

The effects of exercise on insulin signaling and neuroinflammation are truly relevant in the hippocampus. Physical training reduces the expression of $\beta$-amyloid (34) and tau phosphorylation $^{13}$, classic hallmarks in development of AD. The increased signal transduction of the insulin pathway of key proteins in the hippocampus, as Akt and ERK, is an indication that there is direct positive effect on neuronal survival. Furthermore, the insulin signaling acts on inflammation, reducing the JNK phosphorylation, NF-kB activation and transcription of both pro-inflammatory and pro-apoptotic proteins. For all these actions, it is possible that the exercise is still an underestimated method, but promising method for prevention and treatment of $\mathrm{AD}$. In the same context, Figure 1 shows an original and schematic illustration of the effects of exercise, which promote neuroprotection in the hippocampus.

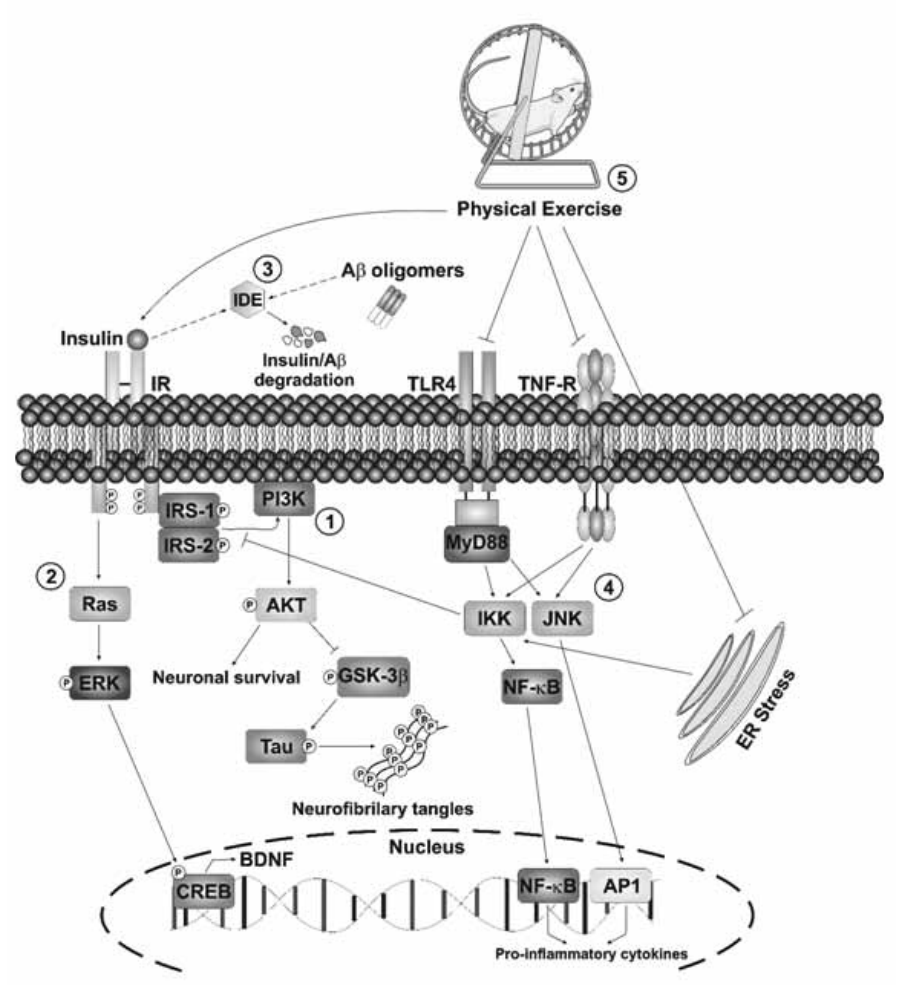

Figure 1. In hippocampal neurons, binding of insulin to the IR active routes of PI3-K/Akt and MAPKs. 1) PI3K/Akt activation leads to inhibition of GSK3 beta activity, reducing phosphorylation of tau protein, a protein strictly involved in AD. This pathway also promotes neuronal survival by an antiapoptotic effect through FOXO phosphorylation and deactivation. FOXO is an important transcriptional factor of genes involved in apoptosis and it is active when dephosphorylated. 2) The MAPKs pathway (Ras/ERK) induces CREB phosphorylation and activation, increasing the transcription of BDNF, an important growth factor. 3) The IDE is a major degradative enzymes $\beta$-amyloid. Higher levels of circulating insulin competes as substrate for degradation by IDE, favoring the concentration of $\beta$-amyloid. The hyperphosphorylation of tau protein and $\beta$-amyloid accumulation are typical characteristics of brain with Alzheimer's disease. 4) Pro-inflammatory mechanisms, such as activation of JNK and IKK enzymes by TNF- $\alpha$, TLR4 and Endoplasmic Reticulum Stress, reduce the ability of insulin to propagate their intracellular signal and contribute to the pathogenesis of Alzheimer's disease. 5) On the other hand, physical exercise has been shown to be effective in attenuating inflammation and restoring the insulin signaling in hippocampal neurons and proved to be an interesting alternative for the 
treatment of $\mathrm{AD}$. AD, Alzheimer disease; IR, insulin receptor; IDE, insulin degrading enzyme; IRS-1/2, 1/2 subtract insulin receptor; PI3-K, phosphatidylinositol 3 kinase; Akt protein kinase B; GSK3- $\beta$, glycogen synthase kinase 3 beta; ERK, extracellular signal-regulated kinase; CREB: cAMP response element binding protein; BDNF, brain-derived neurotrophic factor TLR4, toll like receptor 4; TNF-R, tumor necrosis factor receptor; IKK, I kappa B kinase; JNK, c-Jun N-terminal kinase; $\mathrm{NF}-\mathrm{kB}$, nuclear factor kappa B; AP1, activator protein 1 transcriptional factor; ER, endoplasmic reticulum. This figure is original and made by the authors with the purpose of illustrate the molecular mechanisms involved in AD.

In a recent meta-analysis ${ }^{39}$, it was estimated that physical exercise could reduce the risk of AD development by up to $45 \%$. Despite the limitations of performing this measurement due to different exercise protocols used, the studies showed that variables related to physical exercise as type, frequency, intensity, duration and progression could produce different physiological and metabolic responses. Although that is very well established that physical exercise promotes beneficial effects in $\mathrm{AD}$, there is no consensus on which dose between volume, intensity or type of exercise is better to treat ${ }^{40}$. Nevertheless, it is accept that both moderated or high-intensity as resistance and strength training promotes neuroprotective effects ${ }^{40}$.

The greater challenge of the scientific community in the future is overcome the difficulties of translation between species in studies of AD. Elucidate the mechanisms involved in neuroprotection mediated by exercise will help our understanding in the $\mathrm{AD}$ and the understanding of each result may provide the missing information that we need to treat or prevent this disease.

\section{Conclusion}

Exercise has been shown to promote neuronal survival and protection, especially in the hippocampus. This important structure responsible for learning and memory functions is severely affected by AD. The positive effects of exercise are known to reduce low-grade inflammation, increase insulin sensitivity and augment the expression of growth factors in hippocampal neurons.

\section{References}

1. Gomes RJ, de Oliveira CAM, Ribeiro C, Mota CS de A, Moura LP, Tognoli LMMC, et al. Effects of exercise training on hippocampus concentrations of insulin and IGF-1 in diabetic rats. HPC. 2009 Oct;19(10):981-7.

2. De Felice FG, Ferreira ST. Inflammation, defective insulin signaling, and mitochondrial dysfunction as common molecular denominators connecting type 2 diabetes to Alzheimer disease. Diabetes. 2014 Jul;63(7):2262-72.

3. Alzheimer's Disease International. World Alzheimer Report 2015: The Global Impact of Dementia [Internet]. 2015 [cited 2016 Jul 9]. Available from: https:/www.alz.co.uk/research/ WorldAlzheimerReport2015.pdf
4. Intlekofer KA, Cotman CW. Exercise counteracts declining hippocampal function in aging and Alzheimer's disease. Neurobiol Dis. 2013;57:47-55.

5. Brookmeyer R, Johnson E, Ziegler-Graham K, Arrighi HM. Forecasting the global burden of Alzheimer's disease. Alzheimers Dement. Elsevier; 2007 Jul;3(3):186-91.

6. De Felice FG, Munoz DP. Opportunities and challenges in developing relevant animal models for Alzheimer's disease. Ageing Res Rev. 2016;26:112-4.

7. Lovatel GA, Elsner VR, Bertoldi K, Vanzella C, Moysés F dos S, Vizuete A, et al. Treadmill exercise induces age-related changes in aversive memory, neuroinflammatory and epigenetic processes in the rat hippocampus. Neurobiol Learn Mem. 2013;101:94-102.

8. Pauli JR, Cintra DE, de Souza CT, Ropelle ER. Novos mecanismos pelos quais o exercício físico melhora a resistência à insulina no músculo esquelético. Arq Bras Endocrinol e Metab. 2009;53(4):399-408.

9. de la Monte SM, Wands JR. Alzheimer's disease is type 3 diabetes-evidence reviewed. J Diabetes Sci Technol. 2008 Nov;2(6):1101-13.

10. 10. Wang H, Quirion R, Little PJ, Cheng Y, Feng Z-P, Sun H-S, et al. Forkhead box $\mathrm{O}$ transcription factors as possible mediators in the development of major depression. Neuropharmacology. 2015;99:527-37.

11. Osenkowski P, Ye W, Wang R, Wolfe MS, Selkoe DJ. Direct and Potent Regulation of -Secretase by Its Lipid Microenvironment. J Biol Chem. American Society for Biochemistry and Molecular Biology; 2008 Aug 15;283(33):22529-40.

12. Luo D, Hou X, Hou L, Wang M, Xu S, Dong C, et al. Effect of pioglitazone on altered expression of $A \beta$ metabolism-associated molecules in the brain of fructose-drinking rats, a rodent model of insulin resistance. Eur J Pharmacol. 2011 Aug 16;664(1-3):14-9.

13. 13. Diegues JC, Pauli JR, Luciano E, de Almeida Leme JAC, de Moura LP, Dalia RA, et al. Spatial memory in sedentary and trained diabetic rats: molecular mechanisms. HPC. 2014 Jun;24(6):703-11.

14. Bruel-Jungerman E, Veyrac A, Dufour F, Horwood J, Laroche S, Davis S. Inhibition of PI3K-Akt signaling blocks exercise-mediated enhancement of adult neurogenesis and synaptic plasticity in the dentate gyrus. PLoS One. 2009;4(11):e7901.

15. Blázquez E, Velázquez E, Hurtado-Carneiro V, Ruiz-Albusac JM. Insulin in the brain: its pathophysiological implications for States related with central insulin resistance, type 2 diabetes and Alzheimer's disease. Front Endocrinol (Lausanne). 2014;5:161.

16. Kandel ER, Dudai Y, Mayford MR. The Molecular and Systems Biology of Memory. Cell. 2014;157(1):163-86.

17. Korte M, Schmitz D. Cellular and System Biology of Memory: Timing, Molecules, and Beyond. Physiol Rev. 2016 Apr;96(2):647-93.

18. Mehran AE, Templeman NM, Brigidi GS, Lim GE, Chu K-Y, $\mathrm{Hu} \mathrm{X}$, et al. Hyperinsulinemia drives diet-induced obesity independently of brain insulin production. Cell Metab. 2012 Dec 5;16(6):723-37.

19. Chung HY, Cesari M, Anton S, Marzetti E, Giovannini S, Seo AY, et al. Molecular inflammation: Underpinnings of aging and age-related diseases. Ageing Res Rev. 2009;8(1):18-30. 
20. Giunta B, Fernandez F, Nikolic W V, Obregon D, Rrapo E, Town $\mathrm{T}$, et al. Inflammaging as a prodrome to Alzheimer's disease. $\mathrm{J}$ Neuroinflammation. 2008;5:51.

21. Franceschi C, Bonafè M, Valensin S, Olivieri F, De Luca M, Ottaviani E, et al. Inflamm-aging. An evolutionary perspective on immunosenescence. Ann N Y Acad Sci. 2000 Jun;908:244-54.

22. Velloso LA, Folli F, Saad MJ. TLR4 at the Crossroads of Nutrients, Gut Microbiota, and Metabolic Inflammation. Endocr Rev. 2015 Jun;36(3):245-71.

23. Bomfim TR, Forny-Germano L, Sathler LB, Brito-Moreira J, Houzel J-C, Decker H, et al. An anti-diabetes agent protects the mouse brain from defective insulin signaling caused by Alzheimer's disease- associated A $\beta$ oligomers. J Clin Invest. 2012 Apr;122(4):1339-53.

24. Ma Q-L, Yang F, Rosario ER, Ubeda OJ, Beech W, Gant DJ, et al. Beta-amyloid oligomers induce phosphorylation of tau and inactivation of insulin receptor substrate via c-Jun N-terminal kinase signaling: suppression by omega-3 fatty acids and curcumin. J Neurosci. 2009 Jul 15;29(28):9078-89.

25. Boden G. Endoplasmic reticulum stress: another link between obesity and insulin resistance/inflammation? Diabetes. American Diabetes Association; 2009 Mar;58(3):518-9.

26. Pauli JR, Ropelle ER, Cintra DE, de Souza CT. Efeitos do Exercício Físico na Expressão e Atividade da AMPK $\alpha$ em Ratos Obesos Induzidos por Dieta Rica em Gordura. Rev Bras Med do Esporte. 2009;15(2):98-103.

27. Pauli JR, Ropelle ER, Cintra DE, De Souza CT, da Silva ASR, Moraes JC, et al. Acute exercise reverses aged-induced impairments in insulin signaling in rodent skeletal muscle. Mech Ageing Dev. 2010 May;131(5):323-9.

28. Vaynman S, Ying Z, Gomez-Pinilla F. Hippocampal BDNF mediates the efficacy of exercise on synaptic plasticity and cognition. Eur J Neurosci. 2004 Nov;20(10):2580-90.

29. Gligoroska JP, Manchevska S. The effect of physical activity on cognition - physiological mechanisms. Mater Sociomed. 2012;24(3):198-202.

30. Paillard T. Preventive effects of regular physical exercise against cognitive decline and the risk of dementia with age advancement. Sport Med - open. 1(1):4.

31. Van der Borght K, Kóbor-Nyakas DE, Klauke K, Eggen BJL, Nyakas C, Van der Zee EA, et al. Physical exercise leads to rapid adaptations in hippocampal vasculature: temporal dynamics and relationship to cell proliferation and neurogenesis. HPC. 2009 Oct;19(10):928-36.

32. Nokia MS, Lensu S, Ahtiainen JP, Johansson PP, Koch LG, Britton SL, et al. Physical exercise increases adult hippocampal neurogenesis in male rats provided it is aerobic and sustained. J Physiol. 2016 Apr 1;594(7):1855-73.
33. Lou S, Liu J, Chang H, Chen P. Hippocampal neurogenesis and gene expression depend on exercise intensity in juvenile rats. Brain Res. 2008;1210:48-55.

34. Um H-S, Kang E-B, Koo J-H, Kim H-T, Jin-Lee, Kim E-J, et al. Treadmill exercise represses neuronal cell death in an aged transgenic mouse model of Alzheimer's disease. Neurosci Res. 2011;69(2):161-73.

35. Cai M, Wang H, Li J, Zhang Y-L, Xin L, Li F, et al. The signaling mechanisms of hippocampal endoplasmic reticulum stress affecting neuronal plasticity-related protein levels in high fat diet-induced obese rats and the regulation of aerobic exercise. Brain Behav Immun. 2016; in press.

36. Yau SY, Li A, Hoo RLC, Ching YP, Christie BR, Lee TMC, et al. Physical exercise-induced hippocampal neurogenesis and antidepressant effects are mediated by the adipocyte hormone adiponectin. Proc Natl Acad Sci. National Academy of Sciences; 2014 Nov 4;111(44):15810-5.

37. Marosi K, Bori Z, Hart N, Rga LS, Koltai E, Rad Z, et al. Longterm exercise treatment reduces oxidative stress in the hippocampus of aging rats. Neuroscience. 2012;226:21-8.

38. Vingtdeux V, Giliberto L, Zhao H, Chandakkar P, Wu Q, Simon $\mathrm{JE}$, et al. AMP-activated protein kinase signaling activation by resveratrol modulates amyloid-beta peptide metabolism. J Biol Chem. 2010 Mar 19;285(12):9100-13.

39. Hamer M, Chida Y. Physical activity and risk of neurodegenerative disease: a systematic review of prospective evidence. Psychol Med. 2009 Jan;39(1):3-11.

40. Chen W-W, Zhang X, Huang W-J. Role of physical exercise in Alzheimer's disease. Biomedical Rep. 2016 Apr; 4(4):403-7.

\section{Corresponding author}

José Diego Botezelli

Universidade Estadual de Campinas, Limeira, SP, Brasil

Email: jdbotezelli@outlook.com

Manuscript received on August 26, 2016

Manuscript accepted on October 27, 2016

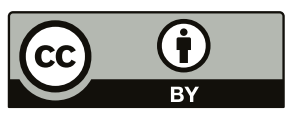

Motriz. The Journal of Physical Education. UNESP. Rio Claro, SP, Brazil - eISSN: 1980-6574 - under a license Creative Commons - Version 3.0 